\title{
PRODUCT FORMULAS FOR VOLUMES OF FLOW POLYTOPES
}

\author{
KAROLA MÉSZÁROS \\ (Communicated by Jim Haglund)
}

\begin{abstract}
Intrigued by the product formula $\prod_{i=1}^{n-2} C_{i}$ for the volume of the Chan-Robbins-Yuen polytope $C R Y_{n}$, where $C_{i}$ is the $i^{\text {th }}$ Catalan number, we construct a family of polytopes $\mathcal{P}_{m, n}$, indexed by $m \in \mathbb{Z}_{\geq 0}$ and $n \in \mathbb{Z}_{\geq 2}$, whose volumes are given by the product$$
\prod_{i=m+1}^{m+n-1} \frac{1}{2 i+1}\left(\begin{array}{c}
m+n+i+1 \\
2 i
\end{array}\right) .
$$

The Chan-Robbins-Yuen polytope $C R Y_{n}$ coincides with $\mathcal{P}_{0, n-2}$. Our construction of the polytopes $\mathcal{P}_{m, n}$ is an application of a systematic method we develop for expressing volumes of a class of flow polytopes as the number of certain triangular arrays. This method can also be used as a heuristic technique for constructing polytopes with combinatorial volumes. As an illustration of this we construct polytopes whose volumes equal the number of $r$-ary trees on $n$ internal nodes, $\frac{1}{(r-1) n+1}\left(\begin{array}{c}r n \\ n\end{array}\right)$. Using triangular arrays we also express the volumes of flow polytopes as constant terms of formal Laurent series.
\end{abstract}

\section{INTRODUCTION}

In this paper we devise an encoding of triangulations for a large class of flow polytopes. Using this encoding, we prove new volume formulas for a family of polytopes, of which, the famous Chan-Robbins-Yuen polytope is a special case. We also use the encoding as a heuristic for constructing polytopes with combinatorial volumes, as well as to shed light on the geometric nature of several intriguing conjectures of Chan, Robbins, and Yuen [Cha-Rob-Yue, which previously had no polytopal interpretations. We tie the whole story in with constant terms of formal Laurent series.

The Chan-Robbins-Yuen polytope $C R Y_{n+2}$ was discovered in 1998 by Chan, Robbins, and Yuen [Cha-Rob-Yue, while they were studying the Birkhoff polytope. (The $C R Y_{n+2}$ polytope is a face of the Birkhoff polytope.) Chan, Robbins, and Yuen [Cha-Rob-Yue] conjectured that the volume of $C R Y_{n+2}$ was $\prod_{i=1}^{n} C_{i}$, where $C_{i}=\frac{1}{i+1}\left(\begin{array}{c}2 i \\ i\end{array}\right)$ is the $i^{t h}$ Catalan number. This conjecture, which thanks to

Received by the editors December 1, 2011 and, in revised form, November 30, 2012 and February $23,2013$.

2010 Mathematics Subject Classification. Primary 05E10, 51M25, 52B11.

Key words and phrases. Flow polytope, Chan-Robbins-Yuen polytope, triangular arrays, triangulation, volume, Kostant partition function.

The author was supported by a National Science Foundation Postdoctoral Research Fellowship (DMS 1103933). 
Zeilberger [Zei] quickly became a theorem, has captivated combinatorialists and noncombinatorialists alike. It continues to intrigue, since no combinatorial proof of it is known.

Without reference to polytopes the above well-known result can be stated as

$$
K_{A_{n}^{+}}\left(1,2, \ldots, n,-\left(\begin{array}{c}
n+1 \\
2
\end{array}\right)\right)=\prod_{k=1}^{n} C_{k}
$$

where $K_{A_{n}^{+}}(\mathrm{v})$ is the Kostant partition function of type $A_{n}$, which is equal to the number of ways to write the vector $\mathrm{v}$ as a nonnegative linear combination of the positive type $A_{n}$ roots without regard to order. While endowed with combinatorial meaning, Kostant partition functions were introduced in and are a vital part of representation theory: weight multiplicities and tensor product multiplicities can be expressed in terms of the Kostant partition function. Kostant partition functions also come up in toric geometry and analytic residue theory.

In their paper Cha-Rob-Yue Chan, Robbins, and Yuen defined the $C R Y_{n+2}$ polytope and constructed a triangulation of it, the simplices of which they bijected with certain triangular arrays. Their triangulation is specific to the $C R Y_{n+2}$ polytope and provokes the question of how the triangular arrays came up. In their famous Conjecture 1, Chan, Robbins, and Yuen stated that the number of the triangular arrays encoding the triangulation of $C R Y_{n+2}$ is $\prod_{i=1}^{n} C_{i}$. It was this form of the conjecture that Zeilberger [Zei] proved in a very elegant, though noncombinatorial manner, using the Morris constant term identity Morr. Chan, Robbins and Yuen [Cha-Rob-Yue, Conjecture 2, 3] gave two other conjectural formulas for the number of certain other triangular arrays, which they did not give polytopal interpretations for and which they mentioned came up in their computational exprimentations. Zeilberger [Zei] showed how to prove those conjectures, too, using the Morris constant term identity Morr. Among other things, in this paper we show that the triangular arrays in Conjectures 2 and 3 of Cha-Rob-Yue can be interpreted in the world of polytopes. Furthermore, we show that triangular arrays in general, of which the ones appearing in Conjectures 1, 2 and 3 of Cha-Rob-Yue are just special cases in disguise, are an essential part of the study of triangulations of flow polytopes.

We develop a systematic method for expressing volumes of a class of flow polytopes as the number of certain triangular arrays. As an application of this method we construct a family of polytopes $\mathcal{P}_{m, n}$, indexed by $m \in \mathbb{Z}_{\geq 0}$ and $n \in \mathbb{Z}_{>0}$, whose normalized volumes are given by the product

$$
\prod_{i=m+1}^{m+n-1} \frac{1}{2 i+1}\left(\begin{array}{c}
m+n+i+1 \\
2 i
\end{array}\right) .
$$

The Chan-Robbins-Yuen polytope $C R Y_{n}$ coincides with $\mathcal{P}_{0, n-2}$. It is not immediate from the formula (2) that the volume of $\mathcal{P}_{0, n-2}$ is $\prod_{i=1}^{n-2} C_{i}$, that of $C R Y_{n}$, but this can be seen using (8). Since the volume of any flow polytope can be expressed as a Kostant partition function evaluated at a vector, our results also imply interesting formulas for special evaluations of the Kostant partition function. Among other identities, we deduce a generalization of (11) using the polytopes $\mathcal{P}_{m, n}$ mentioned 
above:

(3)

$K_{A_{n}^{+}}\left(m+1, m+2, \ldots, m+n,-n m-\left(\begin{array}{c}n+1 \\ 2\end{array}\right)\right)=\prod_{i=m+1}^{m+n-1} \frac{1}{2 i+1}\left(\begin{array}{c}m+n+i+1 \\ 2 i\end{array}\right)$.

Equation (3) has been observed by A.N. Kirillov in a different, but equivalent form, in his amazing paper Kir and a proof of it via residue techniques appeared in [Bal-Ver2, Section 3].

Our method of encoding triangulations as triangular arrays also yields a heuristic technique for constructing polytopes with combinatorial volumes. As an illustration of this we construct polytopes whose volumes equal the number of $r$-ary (ordered) trees on $n$ internal nodes, $\frac{1}{(r-1) n+1}\left(\begin{array}{c}r n \\ n\end{array}\right)$ [Loe, Section 3.13]. We also express the volumes of flow polytopes as constant terms of formal Laurent series.

The outline of this paper is as follows. In Section 2 we provide the basic definitions and background about flow polytopes and Kostant partition functions. In Section 3 we present an algorithm for triangulating flow polytopes and prove that the volume of a flow polytope is equal to the number of certain triangular arrays depending on the polytope. We also express the latter as the constant term of a formal Laurent series. Section 4 is devoted to studying a family of polytopes $\mathcal{P}_{m, n}$, $m \in \mathbb{Z}_{\geq 0}, n \in \mathbb{Z}_{\geq 2}$, of which the Chan-Robbins-Yuen polytope is a special case, and proving that the normalized volume of $\mathcal{P}_{m, n}$ is as given in (2). At the same time we give Cha-Rob-Yue, Conjecture 3] a polytopal interpretation. In Section 5 we provide a polytopal interpretation of [Cha-Rob-Yue, Conjecture 2], thereby constructing collections of polytopes $\left\{P_{i}^{n, k}\right\}_{i \in C_{n, k}}$, for fixed $k \leq n$, where $C_{n, k}$ is the set over which the indices $i$ run, such that the sum of volumes of $P_{i}^{n, k}$, with $k, n$ fixed and $i \in C_{n, k}$ is

$$
N(n, k) \times \prod_{i=1}^{n-1} C_{i}
$$

where

$$
N(n, k)=\frac{1}{n}\left(\begin{array}{l}
n \\
k
\end{array}\right)\left(\begin{array}{c}
n \\
k-1
\end{array}\right)
$$

is the Narayana number. In Section 6 we construct a family of polytopes with volumes equal to the number of $r$-ary trees on $n$ internal nodes, $\frac{1}{(r-1) n+1}\left(\begin{array}{c}r n \\ n\end{array}\right)$, as an illustration of how to use our triangular array encoding for constructing polytopes with combinatorial volumes. Section 7 is meant as some pleasant little fun: we study natural generalizations of the polytope Catalonotope, and calculate their volumes. Postnikov and Stanley were the first to study (an equivalent version of) the Catalanotope and they calculated its volume, the Catalan number; thus, its name.

\section{BACKGROUND INFORMATION}

2.1. Flow polytopes. Flow polytopes are associated to loopless graphs in the following way. Let $G$ be a graph on the vertex set $[n+1]:=\{1,2, \ldots, n+1\}$, and let in $(e)$ denote the smallest (initial) vertex of edge $e$ and fin $(e)$ the biggest (final) vertex of edge $e$. Think of fluid flowing on the edges of $G$ from the smaller to the larger vertices, so that the total fluid volume entering vertex 1 is one and leaving vertex $n+1$ is one, and there is conservation of fluid at the intermediate vertices. 


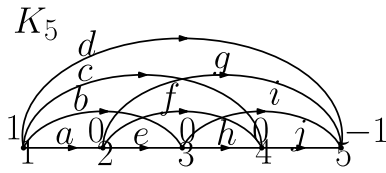

$$
\begin{aligned}
& 1=a+b+c+d \\
& 0=e+f+g-a \\
& 0=h+i-b-e \\
& 0=j-c-f-h
\end{aligned}
$$

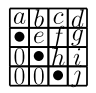

FiguRE 1. Graph $K_{5}$ is given with arrows on its edges suggestive of the direction of the flows. The flow variables on the edges are $a, b, c, d, e, f, g, h, i, j$. Vertices $1,2,3,4,5$ are written below the graph, while the net flow vector $(1,0,0,0,-1)$ is above the vertices. The equations defining the flow polytope corresponding to $K_{5}$ are in the middle. Note that these same equations define $C R Y_{4}$ as can be seen from the matrix on the left, where we denoted by $\bullet$ entries that are determined by the variables $a, b, c, d, e, f, g, h, i, j$.

Formally, a flow $f$ of size one on $G$ is a function $f: E \rightarrow \mathbb{R}_{\geq 0}$ from the edge set $E$ of $G$ to the set of nonnegative real numbers such that

$$
1=\sum_{e \in E, \operatorname{in}(e)=1} f(e)=\sum_{e \in E, \operatorname{fin}(e)=n+1} f(e),
$$

and for $2 \leq i \leq n$

$$
\sum_{e \in E, \operatorname{fin}(e)=i} f(e)=\sum_{e \in E, \operatorname{in}(e)=i} f(e) .
$$

The flow polytope $\mathcal{F}_{G}$ associated to the graph $G=(V, E)$ is the set of all flows $f: E \rightarrow \mathbb{R}_{\geq 0}$ of size one on $G$. The polytope $\mathcal{F}_{G}$ is a convex polytope in the Euclidean space $\mathbb{R}^{|E|}$ of all functions $f: E \rightarrow \mathbb{R}$. Its $\operatorname{dimension}$ is $\operatorname{dim}\left(\mathcal{F}_{G}\right)=$ $|E|-|V|+1$ Bal-Ver2. Figure 1 shows the equations of $\mathcal{F}_{K_{5}}$ and explains why this polytope is the same as $C R Y_{4}$. The same argument can be used to show that $\mathcal{F}_{K_{n+1}}$ and $C R Y_{n}$ coincide. Note that $K_{n}$ denotes the complete graph on $n$ vertices. In their unpublished works Pos, Sta1 Postnikov and Stanley discovered a remarkable connection between the volume of the flow polytope and the Kostant partition function $K_{G}$. Namely, they proved that given a loopless graph $G$ on the vertex set $[n+1]$, the normalized volume $\operatorname{vol}\left(\mathcal{F}_{G}\right)$ of the flow polytope associated to graph $G$ is

$$
\operatorname{vol}\left(\mathcal{F}_{G}\right)=K_{G}\left(0, d_{2}, \ldots, d_{n},-\sum_{i=2}^{n} d_{i}\right),
$$

where $d_{i}=\operatorname{indeg}_{G}(i)-1$ for $i \in\{2, \ldots, n\}$ (indeg ${ }_{G}(i)$ denotes the in degree of vertex $i$ in graph $G$ ), and $K_{G}(\mathrm{v})$ denotes the Kostant partition function, which is the number of ways to write the vector $\mathrm{v}$ as a nonnegative linear combination of the positive type $A_{n}$ roots corresponding to the edges of $G$, without regard to order. To the edge $(i, j), i<j$, of $G$ corresponds the positive type $A_{n}$ root $e_{i}-e_{j}$, where $e_{i}$ is the $i^{\text {th }}$ standard basis vector in $\mathbb{R}^{n+1}$.

Along with Postnikov and Stanley Pos, Sta1, Baldoni and Vergne Bal-Ver1, Bal-Ver2 also studied type $A_{n}$ flow polytopes extensively with residue techniques. The author and Morales [Mész-Mora worked on flow polytopes of types $C_{n}$ and $D_{n}$ using combinatorial techniques. 


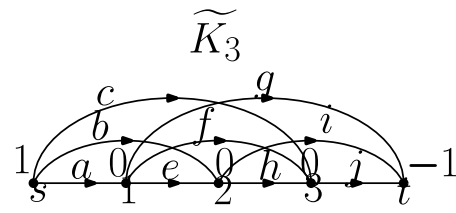

$$
\begin{aligned}
& 1=a+b+c \\
& 0=e+f+g-a \\
& 0=h+i-b-e \\
& 0=j-c-f-h
\end{aligned}
$$

FIGURE 2. Graph $\widetilde{K_{3}}$ and the equations defining the flow polytope $\mathcal{F}_{\widetilde{K_{3}}}$.

2.2. Triangulation of flow polytopes. We show a systematic way of triangulating flow polytopes $\mathcal{F}_{G}$ associated to graphs $G$ with special source and sink vertices. This process was used by Pos, Sta1] in their unpublished works and appears in its most general form in Mész-Mora. A related process has been studied in detail in the context of root polytopes by the author Mész1, Mész2.

Let $G=([n+1], E)$ and let $\widetilde{G}=([n+1] \cup\{s, t\}, \widetilde{E})$, where $s$ is the smallest, $t$ is the largest vertex of $[n+1] \cup\{s, t\}$, and $\widetilde{E}=E \cup\{(s, i),(i, t) \mid i \in[n+1]\}$. Figure 2 shows an example of the flow polytope $\mathcal{F}_{\widetilde{K_{3}}}$. While by definition $\widetilde{G}$ does not have any edges between $s$ and $t$, it will become apparent that the normalized volume of $\widetilde{G}$ and a graph that is obtained from $\widetilde{G}$ by adding any number of edges between $s$ and $t$ are the same. As such the flow polytope of the complete graph $K_{n+1}$, which is also $C R Y_{n}$, fits in this framework.

Algorithmic step: Given a graph $G_{0}$ on the vertex set $[n+1]$ and $(i, j),(j, k) \in$ $E\left(G_{0}\right)$ for some $i<j<k$, let $G_{1}$ and $G_{2}$ be graphs on the vertex set $[n+1]$ with edge sets

$$
\begin{aligned}
& E\left(G_{1}\right)=E\left(G_{0}\right) \backslash\{(j, k)\} \cup\{(i, k)\}, \\
& E\left(G_{2}\right)=E\left(G_{0}\right) \backslash\{(i, j)\} \cup\{(i, k)\} .
\end{aligned}
$$

We say that $G_{0}$ reduces to $G_{1}$ and $G_{2}$ under the reduction rules defined by equations (5). A reduction tree $\mathcal{T}(G)$ of a graph $G$ is defined as follows. The root of $\mathcal{T}(G)$ is labeled by $G$. Each node $G_{0}$ in $\mathcal{T}(G)$ has zero or two children, depending on whether $G_{0}$ can be reduced. If yes, then the two children depend on the choice of the edges of $G_{0}$ on which we perform the reduction. Namely, if the reduction is performed on edges $(i, j),(j, k) \in E\left(G_{0}\right), i<j<k$, then the two children of the node $G_{0}$ are labeled by the graphs $G_{1}$ and $G_{2}$ as described by equation (5). For an example of a reduction tree see Figure 3 . Note that the reduction tree of $G$ is not unique: it depends on the particular reductions we choose at each graph. However, the number of leaves (nodes of the reduction tree which have zero children) of all reduction trees of $G$ is the same, and is the value of the normalized volume of $\mathcal{F}_{\widetilde{G}}$ as Corollary 2 states below.

Proposition 1 ([्Pos, Sta1 $])$. Given a graph $G_{0}$ on the vertex set $[n+1]$ and $(i, j),(j, k) \in E\left(G_{0}\right)$, for some $i<j<k$, let $G_{1}$ and $G_{2}$ be as in equations (5). Then

$$
\mathcal{F}_{\widetilde{G}_{0}}=\mathcal{F}_{\widetilde{G}_{1}} \bigcup \mathcal{F}_{\widetilde{G}_{2}} \text { and } \mathcal{F}_{\widetilde{G}_{1}}^{\circ} \bigcap \mathcal{F}_{\widetilde{G}_{2}}^{\circ}=\emptyset
$$

where $\mathcal{F}_{\widetilde{G}_{0}}, \mathcal{F}_{\widetilde{G}_{1}}, \mathcal{F}_{\widetilde{G}_{2}}$ are of the same dimension and $\mathcal{P}^{\circ}$ denotes the interior of $\mathcal{P}$.

For a sketch of the proof of (6) see [Mész-Mora, Proposition 4.1], and in particular Mész-Mora, Figure $5(\mathrm{R} 1)]$. The statement about the dimensions in Proposition 1 holds since $\operatorname{dim}\left(\mathcal{F}_{G}\right)=|E(G)|-|V(G)|+1$ [Bal-Ver2]. Note that if we add the 


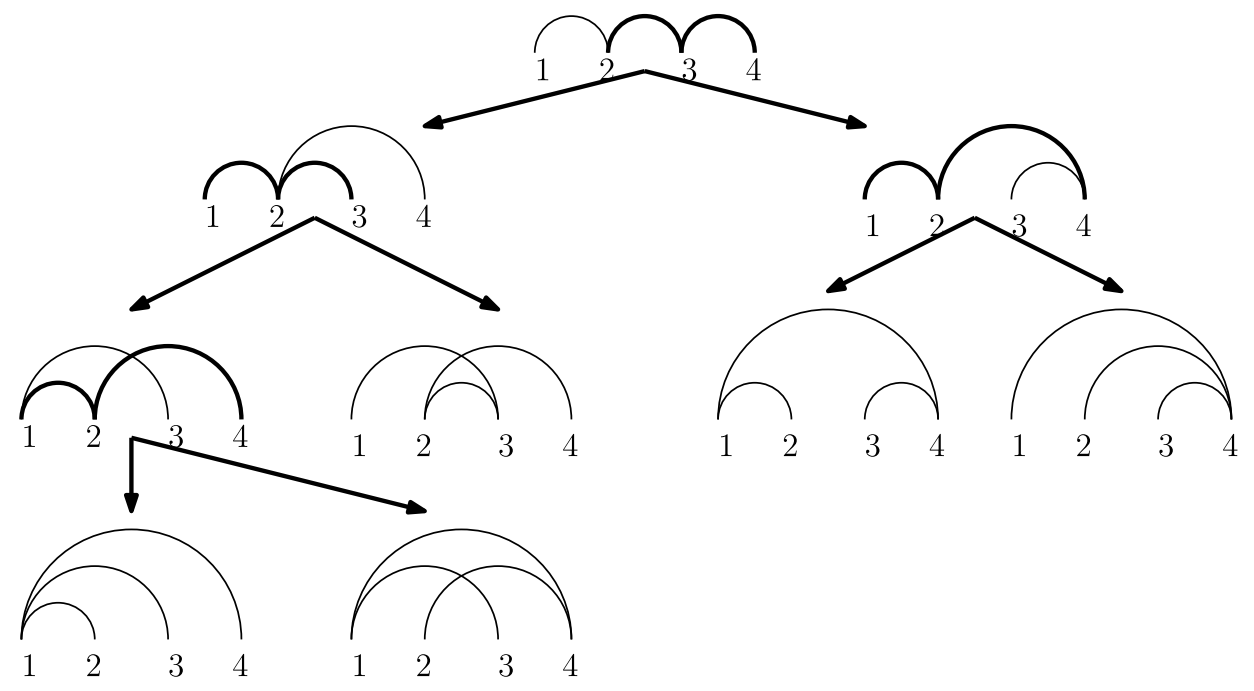

Figure 3. A reduction tree of $G=([4],\{(1,2),(2,3),(3,4)\})$ with five leaves. The edges on which the reductions are performed are in bold.

same number of edges $(s, t)$ to each of $\widetilde{G_{0}}, \widetilde{G_{1}}$ and $\widetilde{G_{2}}$ the statement of Proposition 1 stays the same.

Corollary 2. The normalized volume of $\mathcal{F}_{\widetilde{G}}$ is equal to the number of leaves in a reduction tree $\mathcal{T}(G)$.

\section{VOlumes OF FLOW POLYTOPES AND TRIANGUlar ARRAYS}

In this section we encode the leaves of a reduction tree $\mathcal{T}(G)$ in terms of triangular arrays. In light of Corollary 2 , the calculation of the volume of $\mathcal{F}_{\widetilde{G}}$ is then a matter of enumerating these triangular arrays. We also show that the volume of $\mathcal{F}_{\widetilde{G}}$ is equal to the constant term of a formal Laurent series.

The basic idea is to encode the number of incoming edges of the vertices. We illustrate this idea on a graph $G$ on the vertex set $[b+2]$ with edges $(i, b+1), i \in[b]$, and $(b+1, b+2)$. Figure 4 shows the graphs labeling the leaves of the reduction tree of $G$ for $b=4$. Note that the pair (indeg $(b+1)$, indeg $(b+2)$ ) uniquely determines any leaf of the reduction tree and it takes each value $(b+1-i, i), i \in[b+1]$, exactly once.

The above simple example is the cornerstone of encoding the leaves of a reduction tree of any graph where the only possible multiple edges are of the form $(1, l)$. The case where multiple edges are allowed in general, can be dealt with similarly, and Lemma 18 is the cornerstone of that case. For simplicity, in this paper we mostly consider the case where possible multiple edges are of the form $(1, l)$. For such a case the next lemma is key.

Lemma 3. Given a graph $G$ and a distinguished vertex $v$ in it with $b$ incoming edges and one outgoing edge $(v, u)$, there is a way to perform all reductions possible which involve only edges incident to $v$, so that the leaves of the reduction tree rooted at $G$ are labeled by graphs $G_{i}, i \in[b+1]$, satisfying $\left(\operatorname{indeg}_{G_{i}}(v), \operatorname{indeg}_{G_{i}}(u)\right)=(b+1-i, i)$. 


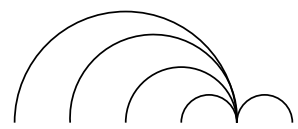

Graph $G$ with $(\operatorname{indeg}(5), \operatorname{indeg}(6))=(4,1)$

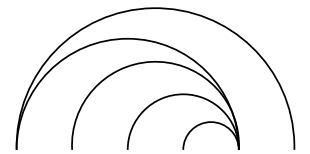

$(\operatorname{indeg}(5), \operatorname{indeg}(6))=(4,1)$

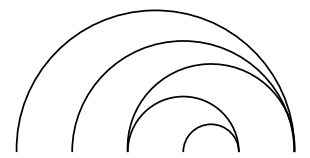

$(\operatorname{indeg}(5), \operatorname{indeg}(6))=(2,3)$

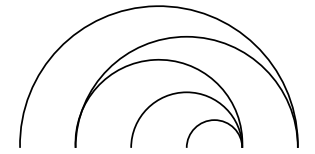

$(\operatorname{indeg}(5), \operatorname{indeg}(6))=(3,2)$

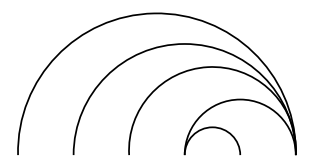

$(\operatorname{indeg}(5), \operatorname{indeg}(6))=(1,4)$

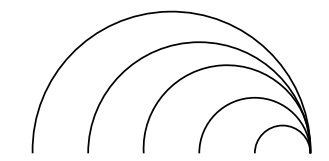

$(\operatorname{indeg}(5), \operatorname{indeg}(6))=(0,5)$

FiguRE 4. Graph $G$ and the graphs labeling the leaves of its reduction tree.

The proof of Lemma 3 is straightforward if we only use reductions that involve the edge $(w, v)$ with $w$ as small as possible.

Given a graph $G$ on the vertex set $[n+1]$, associate to it its indegree sequence

$$
\operatorname{indeg}(G)=(\operatorname{indeg}(2), \ldots, \operatorname{indeg}(n+1)) .
$$

For notational convenience we also define the $m$-indegree sequence of $G, m \in$ $\mathbb{Z}_{\geq 2}$, to be

where

$$
\operatorname{indeg}^{m}(G)=(\operatorname{indeg}(2), \ldots, \operatorname{indeg}(m)),
$$

$$
\operatorname{indeg}(k)=0 \text { for } k>n+1 \text {. }
$$

We recursively characterize the indegree sequences of the leaves of a particular reduction tree of $G$. We now describe our procedure for doing the reductions.

Denote by $I_{i}$ the set of incoming edges into vertex $i$ in the graph $G$. Denote by $V_{i}$ the vertices $k<i$ such that $(k, i)$ is an edge of $G$. Denote by $G[i]$ the restriction of $G$ to the vertex set $[i]$. Denote by $\operatorname{InSeq}(\mathcal{T}(G))$ the multiset of indegree sequences of the graphs labeling the leaves of the reduction tree $\mathcal{T}(G)$, and by $\operatorname{InSeq}^{m}(\mathcal{T}(G))$ the multiset of $m$-indegree sequences of the graphs labeling the leaves of the reduction tree $\mathcal{T}(G)$.

Algorithm 1. Recursive description of $\operatorname{InSeq}(\mathcal{T}(G))$ :

- Construct the reduction tree $\mathcal{T}(G[2])$. Its only leaf is $G[2]$; thus,

$$
\operatorname{InSeq}(\mathcal{T}(G[2]))=\{(\operatorname{indeg}(G[2]))\}=\{(|E(G[2])|)\} .
$$

- Having constructed $\mathcal{T}(G[i])$, construct the reduction tree $\mathcal{T}(G[i+1])$ from $\mathcal{T}(G[i])$ by appending the vertex $i+1$ and the edges $I_{i+1}$ to all graphs in 
$\mathcal{T}(G[i])$, and then performing reductions at each vertex in $V_{i+1}$ on the graphs corresponding to the leaves of $\mathcal{T}(G[i])$, as described below.

- Let $V_{i+1}=\left\{i_{1}<i_{2}<\cdots<i_{k}\right\}$ and let $\left(s_{2}, \ldots, s_{n+1}\right)$ be one of the sequences in $\operatorname{InSeq}^{n+1}(\mathcal{T}(G[i]))$. Applying Lemma 3 a total of $k$ times to the vertices $i_{1}, \ldots, i_{k}$, in this order, we see that the leaves of $\mathcal{T}(G[i+1])$ which are descendents of the graph which has $(n+1)$-indegree sequence $\left(s_{2}, \ldots, s_{n+1}\right)$ have $(n+1)$-indegree sequences

$$
\left(s_{2}, \ldots, s_{n+1}\right)+v^{i+1}\left[i_{1}\right]+\cdots+v^{i+1}\left[i_{k}\right],
$$

where

$$
\begin{gathered}
v^{i+1}\left[i_{l}\right] \in\left\{\left(c_{2}, \ldots, c_{n+1}\right) \mid c_{i}=0 \text { for } i \in[n+1] \backslash\left\{i_{l}, i+1\right\}, c_{i_{l}}=s_{i_{l}}+1-s,\right. \\
\left.c_{i+1}=s, \text { for } s \in\left[s_{i_{l}}+1\right]\right\}, \text { for } l \in[k] .
\end{gathered}
$$

The above procedure yields a complete description of the set $\operatorname{InSeq}(\mathcal{T}(G))$ using the sets $V_{i}, i \in[n+1]$. In order to calculate the volume of $\mathcal{F}_{\widetilde{G}}$ we need to evaluate $|\operatorname{InSeq}(\mathcal{T}(G))|$, and Algorithm 1 imples the following theorem.

Theorem 4. The normalized volume $\operatorname{vol}\left(\mathcal{F}_{\widetilde{G}}\right)$, where $G$ is a graph on the vertex set $[n+1]$, is equal to the number of indegree sequences of the graphs labeling the leaves of the reduction tree $\mathcal{T}(G)$ :

$$
\operatorname{vol}\left(\mathcal{F}_{\widetilde{G}}\right)=|\operatorname{InSeq}(\mathcal{T}(G))|
$$

Furthermore, $|\operatorname{InSeq}(\mathcal{T}(G))|$ can be characterized as follows. Let $\left(a_{i, 1}, \ldots, a_{i, i}\right)$ be the indegree sequences of $\mathcal{T}(G[i+1]), i \in[n]$, as defined above. Then,

- $a_{1,1}=|E(G[2])|$.

- $a_{i, j} \in\left\{0, \ldots, a_{i-1, j}\right\}$, if $(j+1, i+1) \in E(G)$.

- $a_{i, j}=a_{i-1, j}$, if $(j+1, i+1) \notin E(G)$.

- $a_{i, i}=|E(G[i+1])|-\sum_{k=1}^{i-1} a_{i, k}$.

In the above, and below, $a_{i, j}$ denotes the variable for the indegree of vertex $j+1$ in the graph on $i+1$ vertices. The variables $\left(a_{i, j}\right)_{i>j}, j \in[n-1], i \in\{j+1, \ldots, n\}$, can be arranged in a triangular array with the second index of the array fixed in each row and increasing in columns and the first index decreasing in the rows and fixed in the columns.

Example. For $G=([5],\{(1,2),(1,3),(1,5),(2,4),(3,5)\})$ the triangular array is

$$
\begin{array}{lll}
a_{4,1} & a_{3,1} & a_{2,1} \\
a_{4,2} & a_{3,2} \\
a_{4,3} &
\end{array}
$$

subject to the constraints

$$
\begin{aligned}
& 0 \leq a_{4,1}=a_{3,1} \leq a_{2,1}=1\left(=a_{1,1}\right), \\
& 0 \leq a_{4,2} \leq a_{3,2}=2-a_{1,1}\left(=a_{2,2}\right), \\
& 0 \leq a_{4,3}=3-a_{3,2}-a_{3,1}\left(=a_{3,3}\right) .
\end{aligned}
$$

Theorem 4 states that $\operatorname{vol}\left(\mathcal{F}_{\widetilde{G}}\right)$ is equal to the number of triangular arrays with the constraints given above. 
Theorem 5. The normalized volume $\operatorname{vol}\left(\mathcal{F}_{\widetilde{G}}\right)$, where $G$ is a graph on the vertex set $[n+1]$, is equal to the number of triangular arrays $\left(b_{i, j}\right)_{i>j}, j \in[n-1], i \in$ $\{j+1, \ldots, n\}$, with the constraints

$$
b_{n, i}+b_{n-1, i}+\cdots+b_{i+1, i} \leq|E(G[i+1])|-|E(G[i])|+\sum_{k=1}^{i-1} b_{i k}, \text { for all } i \in[n-1]
$$

and constraints $b_{j, i}=0$ if $(i+1, j+1) \notin E(G)$.

Proof. Let $b_{i, i}=|E(G[i+1])|-|E(G[i])|+\sum_{k=1}^{i-1} b_{i k}$. It is then not hard to check that the following is a bijection between the arrays $\left(b_{i, j}\right)_{i \geq j}$, and $\left(a_{i, j}\right)_{i \geq j}, j \in[n-1]$, $i \in\{j, \ldots, n\}$ :

- $b_{i, i}=a_{i, i}=|E(G[i+1])|-\sum_{k=1}^{i-1} a_{i k}$.

- $b_{j, i}=a_{j-1, i}-a_{j, i} i+1 \leq j \leq n$.

Note that $b_{j, i}=0$ if $a_{j-1, i}=a_{j, i}$, that is, if $(i+1, j+1) \notin E(G)$.

Theorem 6. The normalized volume $\operatorname{vol}\left(\mathcal{F}_{\widetilde{G}}\right)$, where $G$ is a graph on the vertex set $[n+1]$, is equal to

$$
\begin{aligned}
C T_{x_{n}} \cdots C T_{x_{1}} \prod_{i=2}^{n}\left(1-x_{i}\right)^{-1} \prod_{(i, n+1) \in E(G): 2 \leq i}\left(1-x_{i}\right)^{-1} \prod_{i=2}^{n} x_{i}^{-c_{i-1}} \\
\\
\prod_{(i, j) \in E(G): j \leq n}\left(1-\frac{x_{i}}{x_{j}}\right)^{-1},
\end{aligned}
$$

where $c_{i}=|E(G[i+1])|-|E(G[i])|$, and $C T_{x_{i}}$ mean the constant term in the expansion of the variable $x_{i}$.

Proof. It suffices to show that the number of triangular arrays $\left(b_{i j}\right)_{i>j}$ as described in Theorem [5] corresponding to graph $G$, is equal to the constant term described above.

Given a triangular array $\left(b_{i j}\right)_{i \geq j}$ corresponding to graph $G$ on the vertex set $[n+1]$, define $s_{i}$ by the equation

$$
s_{i}+\sum_{m=i+1}^{n} b_{m, i}=|E(G[i+1])|-|E(G[i])|+\sum_{k=1}^{i-1} b_{i k}, \text { for all } i \in[n-1] .
$$

Also, let $b_{i, 0}=0$ for $i \in[n]$.

Then, the following term of the above product is constant:

$$
\prod_{i=1}^{n} x_{i}^{s_{i-1}} \prod_{(i, n+1) \in E(G)} x_{i}^{b_{n, i-1}} \prod_{i=2}^{n} x_{i}^{-c_{i-1}} \prod_{(i, j) \in E(G): j \leq n}\left(\frac{x_{i}}{x_{j}}\right)^{b_{j-1, i-1}},
$$

since the power of $x_{i+1}, i \geq 1$, in it is $s_{i}+\sum_{m=i+1}^{n} b_{m, i}-|E(G[i+1])|+|E(G[i])|-$ $\sum_{k=1}^{i-1} b_{i k}=0$, and the power of $x_{1}$ is clearly 0 . Conversely, observe that any constant term is of the previous form, proving Theorem 6 . 
Note that the $c_{i}$ 's, $i \in\{2,3, \ldots, n\}$, in Theorem [6 are equal to the indegree of vertex $i+1$ minus one in $\widetilde{G}$. These same indegrees, albeit denoted and indexed differently, appear in equation (44), studied originally by Posnikov and Stanley [Pos, Sta1, and later by Baldoni and Vergne [Bal-Ver1, Bal-Ver2 using residue techniques. The appearance of the indegrees in Theorem 6 is no coincidence: equation (4) can be deduced from Theorem [6] and assuming (4) another proof of Theorem [6 can be obtained.

\section{Polytopes With volume $\prod_{i=m+1}^{m+n-1} \frac{1}{2 i+1}\left(\begin{array}{c}m+n+i+1 \\ 2 i\end{array}\right)$}

In this section we define a family of polytopes $\mathcal{P}_{m, n}, m \in \mathbb{Z}_{\geq 0}, n \in \mathbb{Z}_{\geq 2}$, of which the Chan-Robbins-Yuen polytope is a special case, and prove that the normalized volume of $\mathcal{P}_{m, n}$ is

$$
\prod_{i=m+1}^{m+n-1} \frac{1}{2 i+1}\left(\begin{array}{c}
m+n+i+1 \\
2 i
\end{array}\right)
$$

using our results from the previous section together with a result of Zeilberger's. At the same time we also provide a polytopal interpretation for Conjecture 3 of Chan, Robbins and Yuen Cha-Rob-Yue, Conjecture 3] which was previously given only in terms of triangular arrays. Finally, we prove a Kostant partition function identity as a corollary of our results.

Consider the family of graphs $G_{m, n}, m \in \mathbb{Z}_{\geq 0}$, on the vertex set $[n+1]$ and with multiset of edges containing all edges of the complete graph, where the edges incident to 1 have multiplicity $m+1$. See Figure 5 for $G_{0,5}$ and $G_{2,5}$.
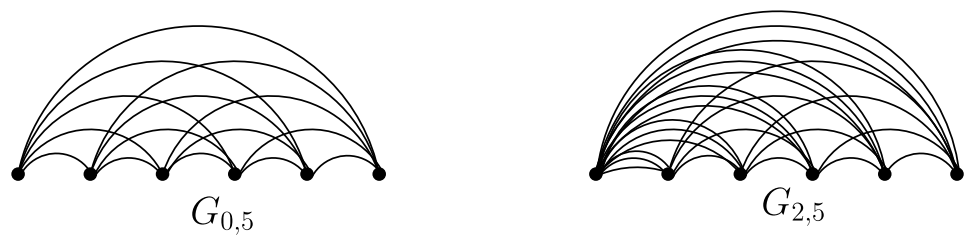

Figure 5. Graphs $G_{0,5}$ and $G_{2,5}$.

By Theorem 4. $\left|\operatorname{InSeq}\left(\mathcal{T}\left(G_{m, n}\right)\right)\right|, m \in \mathbb{Z}_{\geq 0}$, is enumerated by the following triangular arrays. Let $\left(a_{i 1}, \ldots, a_{i i}\right)$ be the indegree sequences of $\mathcal{T}\left(G_{m, n}[i+1]\right)$. Then,

- $a_{11}=m+1$.

- $a_{i j} \in\left\{0, \ldots, a_{i-1, j}\right\}$, for $2 \leq i, 1 \leq j<i$.

- $a_{i i}=\left(\begin{array}{c}i+1 \\ 2\end{array}\right)+i m-\sum_{k=1}^{i-1} a_{i k}$.

We can write the conditions on the $a_{i j}, i, j \in[n], j<i$, in a triangular array. Namely, let the $i^{\text {th }}$ row of the triangular array be

$$
0 \leq a_{n, i} \leq a_{n-1, i} \leq \cdots \leq a_{i+1, i} \leq\left(\begin{array}{c}
i+1 \\
2
\end{array}\right)+i b-\sum_{k=1}^{i-1} a_{i k} .
$$


Example. The triangular array for $n=5$ looks like:

$$
\begin{aligned}
& 0 \leq a_{5,1} \leq a_{4,1} \leq a_{3,1} \leq a_{21} \leq\left(\begin{array}{l}
2 \\
2
\end{array}\right)+m, \\
& 0 \leq a_{5,2} \leq a_{4,2} \leq a_{3,2} \leq\left(\begin{array}{l}
3 \\
2
\end{array}\right)+2 m-a_{21}, \\
& 0 \leq a_{5,3} \leq a_{4,3} \leq\left(\begin{array}{l}
4 \\
2
\end{array}\right)+3 m-a_{31}-a_{32}, \\
& 0 \leq a_{5,4} \leq\left(\begin{array}{l}
5 \\
2
\end{array}\right)+4 m-a_{41}-a_{42}-a_{43} .
\end{aligned}
$$

The following is a special case of Theorem 5 .

Theorem 7. There is a bijection between triangular arrays whose $i^{\text {th }}$ row $a_{n, i}$, $a_{n-1, i}, \cdots, a_{i+1, i}$ with $a_{i j} \geq 0$, satisfies

$$
a_{n, i} \leq a_{n-1, i} \leq \cdots \leq a_{i+1, i} \leq\left(\begin{array}{c}
i+1 \\
2
\end{array}\right)+i m-\sum_{k=1}^{i-1} a_{i k}
$$

and triangular arrays whose $i^{\text {th }}$ row $b_{n, i}, b_{n-1, i}, \cdots, b_{i+1, i}$, with $b_{i j} \geq 0$, satisfies

$$
b_{n, i}+b_{n-1, i}+\cdots+b_{i+1, i} \leq m+i+\sum_{k=1}^{i-1} b_{i k} .
$$

Using Theorem 7 we arrive at the main result of this section, which is a formula for the volume of $\mathcal{P}_{m, n}:=\mathcal{F}_{\widetilde{G}_{m, n}}$ as well as the geometrical realization of Cha-Rob-Yue, Conjecture 3] as the proof of Theorem 8 shows:

Theorem 8. The normalized volume of the flow polytope $\mathcal{P}_{m, n}=\mathcal{F}_{\widetilde{G}_{m, n}}$ is

$$
\prod_{i=m+1}^{m+n-1} \frac{1}{2 i+1}\left(\begin{array}{c}
m+n+i+1 \\
2 i
\end{array}\right)
$$

Proof. By Theorems 4 and 7 , the normalized volume of the flow polytope $\mathcal{F}_{\widetilde{G}_{m, n}}$ is equal to the number of triangular array $\left(b_{i j}\right)_{j<i}$ as described in Theorem 7 . The triangular array $\left(b_{i j}\right)_{j<i}$ agrees with the set of triangular arrays $\mathcal{A}_{m+n+2, m+1}$ described in [Cha-Rob-Yue, Conjecture 3] upon appending $m+1$ rows of 0 's on top. Cha-Rob-Yue, Conjecture 3] states that the number of such arrays is $\prod_{i=m+1}^{m+n-1} \frac{1}{2 i+1}\left(\begin{array}{c}m+n+i+1 \\ 2 i\end{array}\right)$. Zeilberger proved this in [Zei] using the Morris constant term identity.

Theorem 8 implies the Kostant partition function identity stated in Corollary 9 This identity was observed by A.N. Kirillov in a somewhat different form [Kir, p. 80] and was proved using residue techniques in Bal-Ver2, Section 3].

\section{Corollary 9.}

(7)

$$
K_{K_{n+1}}\left(m+1, m+2, \ldots, m+n,-n m-\left(\begin{array}{c}
n+1 \\
2
\end{array}\right)\right)=\prod_{i=m+1}^{m+n-1} \frac{1}{2 i+1}\left(\begin{array}{c}
m+n+i+1 \\
2 i
\end{array}\right) \text {. }
$$


Proof. By (4), Theorem 8 implies

$K_{\widetilde{G}_{m, n}}\left(0,0, m+1, m+2, \ldots, m+n,-n m-\left(\begin{array}{c}n+1 \\ 2\end{array}\right)\right)=\prod_{i=m+1}^{m+n-1} \frac{1}{2 i+1}\left(\begin{array}{c}m+n+i+1 \\ 2 i\end{array}\right)$,

which can be easily seen to be equivalent to (7).

Note that the right hand side of (7) can also be written as

$$
\prod_{p=1}^{n-1} C_{p} \prod_{1 \leq i<j \leq n} \frac{2(m+1)+i+j-1}{i+j-1}
$$

which is the way it appears in [Kir, p. 80]. Here $C_{p}$ is the $p^{\text {th }}$ Catalan number.

\section{A COLlection OF POlytopes Whose Volumes SUM$$
\text { то } N(n, k) \times \prod_{i=1}^{n-1} C_{i}
$$

In this section we construct for each $n, k \in \mathbb{Z}, k \leq n$, a collection of polytopes $\left\{P_{i}^{n, k}\right\}_{i \in C_{n, k}}$, where $C_{n, k}$ is the set over which the indices $i$ run, such that the sum of volumes of $P_{i}^{n, k}$, with $k, n$ fixed and $i \in C_{n, k}$ is

$$
N(n, k) \times \prod_{i=1}^{n-1} C_{i}
$$

where

$$
N(n, k)=\frac{1}{n}\left(\begin{array}{l}
n \\
k
\end{array}\right)\left(\begin{array}{c}
n \\
k-1
\end{array}\right)
$$

is the Narayana number and $C_{i}$ denotes the $i^{\text {th }}$ Catalan number. While curious on its own, we do the above in order to provide a polytopal interpretation of Cha-Rob-Yue, Conjecture 2].

The collections $\left\{P_{i}^{n, k}\right\}_{i \in C_{n, k}}$ are such that for fixed $n$, the polytopes $P_{i}^{n, k}, k=$ $1,2, \ldots, n, i \in C_{n, k}$, are interior disjoint and their union is $\mathcal{F}_{\widetilde{K}_{n+1}}$.

As explained in Section 2, polytopes $C R Y_{n+2}$ and $\mathcal{F}_{K_{n+3}}$ coincide. Moreover, there is a bijection between the triangulations of $\mathcal{F}_{K_{n+3}}$ and the triangulations of $\mathcal{F}_{\widetilde{K}_{n+1}}$ obtained via reductions (5) , since $K_{n+3}$ and $\widetilde{K}_{n+1}$ only defer by one edge which cannot be part of any reduction (see also Proposition 1 and the idea of its proof). In particular the normalized volumes of $\mathcal{F}_{K_{n+3}}$ and $\mathcal{F}_{\widetilde{K}_{n+1}}$ are the same:

$$
\operatorname{vol}\left(\mathcal{F}_{\widetilde{K}_{n+1}}\right)=\prod_{i=1}^{n} C_{i}=\sum_{k=1}^{n} N(n, k) \times \prod_{i=1}^{n-1} C_{i} .
$$

Let the collection $\left\{G_{i}^{n, k}\right\}_{i \in C_{n, k}}$ of graphs be all graphs on the vertex set $[n+1]$ consisting of $\left(\begin{array}{c}n+1 \\ 2\end{array}\right)$ edges, such that

- The edge $(1, n+1)$ has multiplicity $n$,

- all edges other than $(1, n+1)$ have multiplicity zero or one,

- if edge $(1, l), 2 \leq l \leq n$, is in the graph, then edge $(l, n+1)$ is not,

- there are $k-1$ edges of the form $(1, l), 2 \leq l \leq n$, in the graph.

See Figure 6 for an illustration of the graphs $\left\{G_{i}^{3,2}\right\}_{i \in C_{3,2}}$.

Define

$$
P_{i}^{n, k}=\mathcal{F}_{\widetilde{G}_{i}^{n, k}}, \text { for } k \leq n, i \in C_{n, k} .
$$



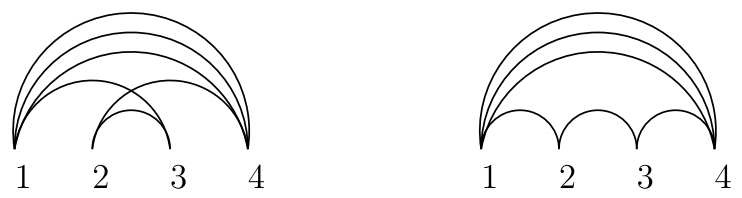

Figure 6. Graphs $\left\{G_{i}^{3,2}\right\}_{i \in C_{3,2}}$.

We set up the definition of $\left\{P_{i}^{n, k}\right\}_{i \in C_{n, k}}$ exactly so that for fixed $n$, the polytopes $P_{i}^{n, k}, k=1,2, \ldots, n, i \in C_{n, k}$, are interior disjoint and their union is $\mathcal{F}_{\widetilde{K}_{n+1}}$.

Proposition 10. For fixed $n$, the polytopes $P_{i}^{n, k}, k=1,2, \ldots, n, i \in C_{n, k}$, are interior disjoint and their union is $\mathcal{F}_{\widetilde{K}_{n+1}}$.

Proof. Note that the graphs $G_{i}^{n, k}, k=1,2, \ldots, n, i \in C_{n, k}$, can be obtained from $K_{n+1}$ by applying the reduction rules as specified in (5) on the edge pairs $(1, l)$, $(l, n+1)$, for each $l \in\{2,3, \ldots, n\}$. Thus, Proposition 11implies Proposition 10 .

Next, we apply Theorem 5 to encode the normalized volumes of the polytopes $P_{i}^{n, k}=\mathcal{F}_{\widetilde{G}_{i}^{n, k}}$, for $k \leq n, i \in C_{n, k}$, by triangular arrays.

Theorem 11. Fix $n, k \in \mathbb{Z}$ such that $1 \leq k \leq n$. Then, the sum of the normalized volumes of the polytopes $P_{i}^{n, k}, i \in C_{n, k}$, is equal to the number of triangular arrays $\left(b_{i, j}\right)_{i>j}, j \in[n-1], i \in\{j+1, \ldots, n\}$, with the constraints

$$
b_{n, i}+b_{n-1, i}+\cdots+b_{i+1, i} \leq n_{i}+\sum_{k=1}^{i-1} b_{i k}, \text { for all } i \in[n-1],
$$

such that $k-1$ of the variables $b_{n, j}, 1 \leq j \leq n-1$ are required to be 0 a priori, and $n_{i}=i$ if $b_{n, i}$ is among these $k$ variables, and $n_{i}=i-1$ otherwise.

Proof. The proof of Theorem 11 is a straightforward application of Theorem 5 for each polytope $P_{i}^{n, k}=\mathcal{F}_{\widetilde{G}_{i}^{n, k}}, i \in C_{n, k}$, involved.

Lemma 12. The triangular arrays $\left(b_{i, j}\right)_{i>j}, j \in[n-1], i \in\{j+1, \ldots, n\}$, with the constraints

$$
b_{n, i}+b_{n-1, i}+\cdots+b_{i+1, i} \leq n_{i}+\sum_{k=1}^{i-1} b_{i k}, \text { for all } i \in[n-1]
$$

such that $k-1$ of the variables $b_{n, j}, 1 \leq j \leq n-1$ are required to be 0 a priori, and $n_{i}=i$ if $b_{n, i}$ is among these $k$ variables, and $n_{i}=i-1$ otherwise, are in bijection with triangular arrays $\left(\widetilde{b}_{i, j}\right)_{i>j}, j \in[n-1], i \in\{j+1, \ldots, n\}$, with the constraints

$$
\widetilde{b}_{n, i}+\widetilde{b}_{n-1, i}+\cdots+\widetilde{b}_{i+1, i} \leq i+\sum_{k=1}^{i-1} \widetilde{b}_{i k}, \text { for all } i \in[n-1]
$$

such that exactly $k-1$ of the inequalities hold at equality.

Proof. Given a triangular array $\left(b_{i, j}\right)_{i>j}, j \in[n-1], i \in\{j+1, \ldots, n\}$, of the first kind with $J \subset[n-1],|J|=k-1$, such that the variables $b_{n, j}, j \in J$, are required to be 0 , let $\left(\widetilde{b}_{i, j}\right)_{i>j}, j \in[n-1], i \in\{j+1, \ldots, n\}$, be the array obtained by setting $\widetilde{b}_{i, j}=b_{i, j}$ for $(i, j) \neq(n, l)$ for $l \in J$. Finally, let $\widetilde{b}_{n, j}=j-\sum_{j+1}^{n-1} \widetilde{b}_{m, j}$, for $j \in J$. 
Note that this way we obtained a triangular array $\left(\widetilde{b}_{i, j}\right)_{i>j}$ for which exactly $k-1$ inequalities hold at equality, namely, the rows indexed by $j \in J$.

Theorem 13. Fix $n, k \in \mathbb{Z}$ such that $1 \leq k \leq n$. Then, the sum of the normalized volumes of the polytopes $P_{i}^{n, k}, i \in C_{n, k}$, is equal to

$$
N(n, k) \times \prod_{i=1}^{n-1} C_{i} .
$$

Proof. Conjecture 2 in the paper of Chan-Robbins-Yuen Cha-Rob-Yue, proved by Zeilberger Zei] enumerates the arrays $\left(\widetilde{b}_{i, j}\right)_{i>j}, j \in[n-1], i \in\{j+1, \ldots, n\}$ as defined in Lemma 12. Thus, Theorem 13 follows from Theorem [11, Lemma 12 together with [Cha-Rob-Yue, Conjecture 2] and its proof in [Zei].

Theorem 13 thus establishes a polytopal realization of Cha-Rob-Yue, Conjecture 2].

\section{Constructing polytopes With volume $\frac{1}{(r-1) n+1}\left(\begin{array}{c}r n \\ n\end{array}\right)$}

In this section we give an example of how to use the triangular arrays to construct polytopes with combinatorial volumes. There is no prescribed formula for this, rather we use the triangular array encoding as stated in Theorem 5 as a heuristic.

Say we would like to construct a family of polytopes with volumes equal the number of $(r+2)$-ary (ordered) trees with $n+1$ internal nodes. If we are to use Theorem 5, this amounts to finding a triangular array $\left(b_{i, j}\right)_{i>j}$ with constraints as described in Theorem 5 and which bijects with $(r+2)$-ary trees with $n+1$ internal nodes. The following proposition takes us close to the solution.

Proposition 14. There is a bijection between nonnegative integer sequences $\left(b_{i+1, i}\right)_{i=1}^{n}$ with constraints $b_{2,1} \leq r+1$ and $b_{i+1, i} \leq r+1+b_{i, i-1}$ for $2 \leq i \leq n$ and $(r+2)$-ary trees with $n+1$ internal nodes.

Proof. Given a nonnegative integer sequences $\left(b_{i+1, i}\right)_{i=1}^{n}$ with constraints $b_{2,1} \leq$ $r+1$ and $b_{i+1, i} \leq r+1+b_{i, i-1}$ for $2 \leq i \leq n$ construct a sequence of rooted ordered $(r+2)$-ary trees $T_{1}, \ldots, T_{n+1}$ as follows. Having constructed $T_{i}, i \in[n]$, we will let $T_{i+1}$ be the tree obtained from $T_{i}$ by marking a leaf of it in a manner described later and adding $r+2$ children to the marked vertex. Let $T_{1}$ be the $(r+2)$-ary tree with $r+3$ vertices consisting of the root $v$ and its children $v_{0}, v_{1}, \ldots, v_{r+1}$ ordered from left to right. This ordered set of vertices of cardinality $r+2$ is denoted by $V_{1}$. Let $V_{i}$ be the set of vertices in $T_{i}$ which are leaves, and such that all vertices which are children of their parents and are to the left of them are also leaves. See Figure 7 for an example. Once the vertices of $V_{i}$ are ordered, and one of its vertices $w$ is marked, we obtain $V_{i+1}$ by deleting the vertices in $V_{i}$ which are children of the parent of $w$ and are equal to $w$ or are to the right of $w$ and adding the $r+2$ children of $w$. The ordering on the vertices which were also in $V_{i}$ is inherited from $V_{i}$, and the $r+2$ new vertices are the last $r+2$ ones and are ordered according to their order from left to right. The marking of a vertex in $V_{i}, i \in[n]$ is done as follows. Mark the $\left(b_{i+1, i}+1\right) s t$ vertex of $V_{i}$. By construction $\left|V_{1}\right|=r+2$ and $\left|V_{i}\right|=r+2+b_{i, i-1}$ for $2 \leq i \leq n$. Thus, the constraints $b_{2,1} \leq r+1$ and $b_{i+1, i} \leq r+1+b_{i, i-1}$ for $2 \leq i \leq n$ can also be written as $b_{2,1}+1 \leq\left|V_{1}\right|$ and $b_{i+1, i}+1 \leq\left|V_{i}\right|$ for $2 \leq i \leq n$. Since the above procedure is clearly invertible, Proposition 14 follows. 


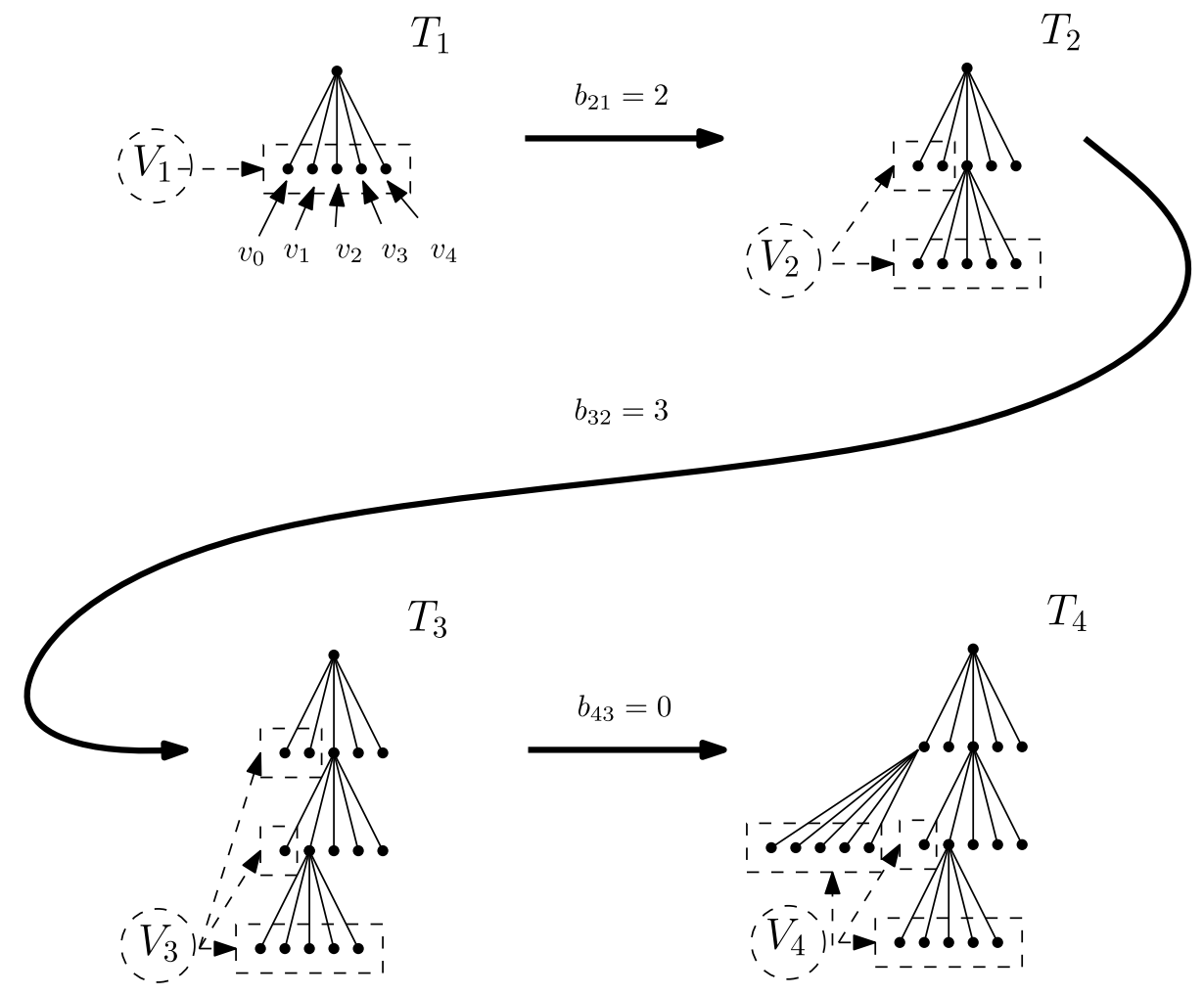

Figure 7. The definitions from the proof of Proposition 14, as well as the construction for $n=3, r=3,\left(b_{21}, b_{32}, b_{43}\right)=(2,3,0)$ are illustrated.

Proposition 15. There is a bijection between nonnegative integer sequences $\left(b_{i+1, i}\right)_{i=1}^{n}$ with constraints $b_{2,1} \leq r+1$ and $b_{i+1, i} \leq r+1+b_{i, i-1}$ for $2 \leq i \leq n$ and the triangular array $\left(b_{i, j}\right)_{i>j}$ with constraints as described in Theorem 5 arising from the graph $G=([n+2], E)$, where the edge set consists of $r+1$ edges $(1,2), r$ edges $(1, i)$, for $i \in\{3,4, \ldots, n+2\}$, and one edge $(i-1, i)$, for $i \in\{3,4, \ldots, n+2\}$.

Proof. The bijection is trivial since for the graph $G$ described above the triangular array $\left(b_{i, j}\right)_{i>j}$ is such that $b_{i, j}=0$ unless $(i, j)=(j+1, j), j \in[n]$, and we have the constraints $b_{2,1} \leq r+1$ and $b_{i+1, i} \leq r+1+b_{i, i-1}$ for $2 \leq i \leq n$.

\section{Corollary 16.}

$$
\operatorname{vol} \mathcal{F}_{\widetilde{G}}=\frac{1}{(r+1)(n+1)+1}\left(\begin{array}{c}
(r+2)(n+1) \\
n+1
\end{array}\right)
$$

where $G$ is as in Proposition 15 above.

Proof. Immediate since the number of $(r+2)$-ary trees on $n+1$ internal vertices is given by $\frac{1}{(r+1)(n+1) n+1}\left(\begin{array}{c}(r+2)(n+1) \\ n+1\end{array}\right)$. 


\section{Corollary 17.}

(10)

$K_{P_{n+2}}(r+1, r+1, \ldots, r+1,-(n+1)(r+1))=\frac{1}{(r+1)(n+1)+1}\left(\begin{array}{c}(r+2)(n+1) \\ n+1\end{array}\right)$,

where $P_{n+2}=([n+2],\{(i, i+1) \mid i \in[n+1]\})$.

Proof. Use equation (4).

\section{The generalized Catalanotope}

The purpose of this section is twofold. On one hand, we study the generalization of the Catalonotope, introduced by Postnikov and Stanley [Pos Sta1] in unpublished works. On the other hand we present Lemma 18 which can be used to encode the triangulations of flow polytopes corresponding to graphs with multiple edges similarly to how we did it above in the case where multiple edges were of the form $(1, l)$.

Postnikov and Stanley Pos, Sta1 studied a root polytope, which they called the Catalonotope in their unpublished works. We consider the flow polytope $\mathcal{F}_{\widetilde{P}_{n+1}}$ corresponding to the path graph $P_{n+1}=([n+1],\{(i, i+1) \mid i \in[n]\})$ a Catalonotope, since the triangulations of this polytope and the root polytope Catalonotope are in bijection. A natural generalization of the Catalonotope is then the flow polytope arising from the graph whose set of edges is the same as that of $P_{n+1}$, but the multiplicities of the edges are not necessarily all 1 .

Denote by $f\left(c_{1}, \ldots, c_{n}\right)$ the volume of $\mathcal{F}_{\widetilde{G}_{c_{1}, \ldots, c_{n}}}$, where $G_{c_{1}, \ldots, c_{n}}$ is a graph on vertex set $[n+1]$ and contains $c_{i}$ edges $(i, i+1)$, for $i \in[n]$. To calculate the volume $f\left(c_{1}, \ldots, c_{n}\right)$, as well as to be able to encode the triangulations of a flow polytope corresponding to a graph with multiple edges, we need the following lemma.

Lemma 18. Let $G$ be the graph on the vertex set [3] with $c_{1}$ edges $(1,2)$ and $c_{2}$ edges $(2,3)$. Then the multiset $\operatorname{InSeq}(G)$ consists of $\left(\begin{array}{c}c_{1}+c_{2}-1-i \\ c_{2}-1\end{array}\right)$ copies of $\left(i, c_{1}+c_{2}-i\right)$, for $i \in\left\{0,1,2, \ldots, c_{1}\right\}$.

The proof of Lemma 18 is routine and is left to the reader.

Then Lemma 18 implies that

$$
f\left(c_{1}, c_{2}, \ldots, c_{n}\right)=\sum_{i=0}^{c_{1}}\left(\begin{array}{c}
c_{1}+c_{2}-1-i \\
c_{2}-1
\end{array}\right) f\left(c_{1}+c_{2}-i, c_{3}, c_{4}, \ldots, c_{n}\right) .
$$

Continually applying Lemma 18 we arrive at the formula

$$
f\left(c_{1}, c_{2}, \ldots, c_{n}\right)=\sum_{\left(k_{2}, \ldots, k_{n}\right) \in S\left(c_{1}, \ldots, c_{n}\right)}\left(\prod_{i=2}^{n-1}\left(\begin{array}{c}
c_{i}-1+k_{i} \\
c_{i}-1
\end{array}\right)\right)\left(\begin{array}{c}
c_{n}+k_{n} \\
c_{n}
\end{array}\right),
$$

where

$$
\begin{gathered}
S\left(c_{1}, \ldots, c_{n}\right)=\left\{\left(k_{2}, \ldots, k_{n}\right) \mid 0 \leq k_{2} \leq c_{1}, 0 \leq k_{i} \leq c_{i-1}+k_{i-1},\right. \\
\text { for } \left.i \in\{3, \ldots, n-1\}, k_{n}=c_{n-1}+k_{n-1}\right\} .
\end{gathered}
$$

We can rewrite equation (11) as stated in the theorem below.

\section{Theorem 19.}

$$
\operatorname{vol} \mathcal{F}_{\widetilde{G}_{c_{1}, \ldots, c_{n}}}=\sum_{\left(k_{2}, \ldots, k_{n}\right) \in S\left(c_{1}, \ldots, c_{n}\right)}\left(\prod_{i=2}^{n-1} \frac{\left(c_{i}\right)^{\left(k_{i}\right)}}{k_{i} !}\right) \frac{\left(c_{n}+1\right)^{\left(k_{n}\right)}}{k_{n} !},
$$


where

$$
(a)^{(b)}:=\prod_{j=0}^{b-1}(a+j)
$$

and $S\left(c_{1}, \ldots, c_{n}\right)$ is as defined above.

\section{Lemma 20.}

$$
\left|S\left(\mathbf{1}_{n}\right)\right|=C_{n},
$$

where $\mathbf{1}_{n}=(1, \ldots, 1) \in \mathbb{Z}^{n}, C_{n}=\frac{1}{n+1}\left(\begin{array}{c}2 n \\ n\end{array}\right)$ denotes the $n^{\text {th }}$ Catalan number.

Proof. Let $f(n)=\left|S\left(\mathbf{1}_{n}\right)\right|$. A sequence $\left(k_{2}, \ldots, k_{n}\right) \in S\left(\mathbf{1}_{n}\right)$ can be thought of as specifying the steps of a walk on the nonnegative $x$-axis. Namely, start at the origin $(0,0)$ and on the $i^{\text {th }}$ step go to $\left(0, k_{i+1}\right), i \in[n-1]$. Write $f(n)=f_{1}(n)+f_{2}(n)$, where $f_{1}(n)$ is the number of sequences $\left(k_{2}, \ldots, k_{n}\right) \in S\left(\mathbf{1}_{n}\right)$ with $k_{2}=0$ and $f_{2}(n)$ is the number of sequences $\left(k_{2}, \ldots, k_{n}\right) \in S\left(\mathbf{1}_{n}\right)$ with $k_{2}=1$. Note that by definition $f_{1}(n)=f(n-1)$. Consider a sequence $s=\left(k_{2}, \ldots, k_{n}\right) \in S\left(\mathbf{1}_{n}\right)$ with $k_{2}=1$. Let the $(i+1)^{\text {st }}$ step be the first one where we are back to the origin; that is, $k_{i+2}=0$ and $k_{j}>0, i+2>j, i>0$. This means that the sequence $\left(k_{3}-1, k_{4}-1, \ldots k_{i+1}-1\right)$ is in $S\left(\mathbf{1}_{i}\right)$ and $\left(k_{i+3}, k_{i+2}, \ldots k_{n}\right)$ is in $S\left(\mathbf{1}_{n-i-1}\right)$. Thus,

$$
f(n)=\sum_{i=0}^{n-1} f(i) f(n-i-1)
$$

where we set $f(0)=1$. Since the Catalan numbers $C_{n}$ satisfy the same relation and the starting value is the same, it follows that $f(n)=C_{n}$.

Corollary 21 ([Pos, Sta1]).

$$
\operatorname{vol}\left(\mathcal{F}_{\widetilde{P}_{n+1}}\right)=C_{n}
$$

where $P_{n+1}=([n+1],\{(i, i+1) \mid i \in[n]\})$.

Proof. Follows immediately from the above, since $f\left(\mathbf{1}_{n}\right)=\left|S\left(\mathbf{1}_{n}\right)\right|=C_{n}$.

Stanley Sta2 pointed out the vague resemblance of (12) to the volume of the Stanley-Pitman polytope Sta-Pit]:

$$
V\left(x_{1}, \ldots, x_{n}\right)=\sum_{k \in S(n)} \prod_{i=1}^{n} \frac{x_{i}^{k_{i}}}{k_{i} !}
$$

where

$$
S(n):=\left\{\boldsymbol{k} \in \mathbb{N}^{n}: \sum_{i=1}^{j} k_{i} \geq j \text { for all } 1 \leq i \leq n-1 \text { and } \sum_{i=1}^{n} k_{i}=n\right\}
$$

with $\mathbb{N}:=\{0,1,2, \ldots\}$.

Is there a connection between the two polytopes that makes this resemblance precise and endows it with a meaning?

\section{ACKNOWLEDGEMENTS}

The author would like to thank Richard Stanley, Alex Postnikov and Alejandro Morales for numerous conversations on the topic of flow polytopes and Kostant partition functions over the course of the past several years. The author would also like to thank the anonymous referee for a careful reading and suggestions. 


\section{REFERENCES}

[Bal-Ver1] Michèle Vergne, Residue formulae for volumes and Ehrhart polynomials of convex polytopes, http://arxiv.org/abs/math/0103097.

[Bal-Ver2] Welleda Baldoni and Michèle Vergne, Kostant partitions functions and flow polytopes, Transform. Groups 13 (2008), no. 3-4, 447-469, DOI 10.1007/s00031-0089019-8. MR 2452600 (2009k:52029)

[Cha-Rob-Yue] Clara S. Chan, David P. Robbins, and David S. Yuen, On the volume of a certain polytope, Experiment. Math. 9 (2000), no. 1, 91-99. MR.1758803 (2001d:52022)

[Kir] Anatol N. Kirillov, Ubiquity of Kostka polynomials, Physics and combinatorics 1999 (Nagoya), World Sci. Publ., River Edge, NJ, 2001, pp. 85-200, DOI 10.1142/9789812810199_0006. MR1865038(2003g:05132)

[Loe] Nicholas A. Loehr, Bijective combinatorics, Discrete Mathematics and its Applications (Boca Raton), CRC Press, Boca Raton, FL, 2011. MR2777360 (2012d:05002)

[Mész1] Karola Mészáros, Root polytopes, triangulations, and the subdivision algebra. I, Trans. Amer. Math. Soc. 363 (2011), no. 8, 4359-4382, DOI 10.1090/S0002-99472011-05265-7. MR2792991 (2012g:52022)

[Mész2] Karola Mészáros, Root polytopes, triangulations, and the subdivision algebra, II, Trans. Amer. Math. Soc. 363 (2011), no. 11, 6111-6141, DOI 10.1090/S0002-99472011-05371-7. MR2817421 (2012g:52021)

[Mész-Mora] Karola Mészáros and Alejandro H. Morales, Flow polytopes and the Kostant partition function, http://arxiv.org/abs/1208.0140.

[Morr] W. G. Morris, Constant Term Identities for Finite and Affine Root Systems: Conjectures and Theorems, Ph.D. Thesis, 1982.

[Pos] A. Postnikov, personal communication, 2010.

[Sta1] Richard P. Stanley, Acyclic flow polytopes and Kostant's partition function, Conference transparencies, 2000, http://math.mit.edu/ rstan/trans.html.

[Sta-Pit] Richard P. Stanley and Jim Pitman, A polytope related to empirical distributions, plane trees, parking functions, and the associahedron, Discrete Comput. Geom. 27 (2002), no. 4, 603-634, DOI 10.1007/s00454-002-2776-6. MR1902680 (2003e:52017)

[Sta2] Richard P. Stanley, personal communication, 2008.

[Zei] Doron Zeilberger, Proof of a conjecture of Chan, Robbins, and Yuen, Orthogonal polynomials: numerical and symbolic algorithms (Leganés, 1998). Electron. Trans. Numer. Anal. 9 (1999), 147-148 (electronic). MR1749805 (2001b:05015)

Department of Mathematics, Cornell University, Ithaca, New York 14853 